\title{
Burden of Illness Among People with Migraine and $\geq 4$ Monthly Headache Days While Using Acute and/or Preventive Prescription Medications for Migraine
}

\author{
Dawn C. Buse, PhD; Marianna S. Yugrakh, MD; Lulu K. Lee, PhD; Jvawnna Bell, MPH, MBA; \\ Joshua M. Cohen, MD, MPH, FAHS; and Richard B. Lipton, MD
}

\begin{abstract}
BACKGROUND: Migraine is a chronic disease that reduces health-related quality of life. Little is known about the burden of migraine in individuals who are potential candidates for preventive treatment with $\geq 4$ monthly headache days currently using migraine medications.

OBJECTIVE: To characterize the burden of migraine among patients reporting $\geq 4$ monthly headache days while taking acute and/or preventive migraine medications.

METHODS: In this retrospective, cross-sectional study, data from the 2016 U.S. National Health and Wellness Survey $(\mathrm{N}=97,503)$ compared the burden of migraine among individuals self-reporting a diagnosis of migraine by a health care professional and $\geq 4$ monthly headache days while using acute and/or preventive prescription migraine medications to matched nonmigraine controls. Propensity score matching across different variables (e.g., age, gender, and body mass index) was used to identify matched controls from respondents who did not self-report a diagnosis of migraine. Migraine-associated burden was measured by impairment in work productivity and daily activities (Work Productivity and Activity Impairment questionnaire), all-cause health care resource utilization (HRU), and allcause direct and indirect costs.
\end{abstract}

RESULTS: This analysis included 197 treated migraine patients with $\geq 4$ monthly headache days and 197 matched nonmigraine controls. Greater proportions of treated migraine patients reported comorbid depression ( $58.4 \%$ vs. $27.9 \%, P<0.001)$ or generalized anxiety disorder (15.2\% vs. $8.6 \%, P=0.043)$ and were on long-term disability $(13.7 \%$ vs. $5.6 \%, P=0.003)$. Absenteeism (11.8\% vs. $6.3 \%, P=0.030)$; presenteeism ( $36.0 \%$ vs. $17.5 \%, P<0.001)$; overall work impairment $(41.0 \%$ vs. $20.9 \%$, $P<0.001)$; and activity impairment $(45.4 \%$ vs. $25.4 \%, P<0.001)$ were greater in treated migraine patients versus nonmigraine controls. Treated migraine patients had higher all-cause HRU and higher all-cause direct $(\$ 24,499.90$ vs. $\$ 15,318.91, P=0.013)$ and indirect $(\$ 14,770.57$ vs. $\$ 5,764.93, P<0.001)$ costs than nonmigraine controls.

CONCLUSIONS: Treated migraine patients with $\geq 4$ monthly headache days reported significantly reduced work productivity and increased all-cause $\mathrm{HRU}$ and cost despite migraine treatment compared with nonmigraine controls. These findings highlight unmet needs in the treatment and management of migraine.

J Manag Care Spec Pharm. 2020;26(10):1334-43

Copyright $\odot 2020$, Academy of Managed Care Pharmacy. All rights reserved.

\section{What is already known about this subject}

Migraine treatment guidelines recommend acute medications for individuals with mild to moderate migraine and migraine-specific acute medications (e.g., triptans and ergotamine derivatives) for those with moderate to severe or difficult-to-treat migraine. Individuals with migraine with $\geq 4$ headache days per month may experience a variety of treatment benefits from migraine preventive therapies according to a recent position statement by the American Headache Society and the results of clinical trials. Increased direct costs (resulting from increased use of medication and diagnostic testing, as well as more frequent visits to health care professionals, the emergency department, and/or hospitalizations) and indirect costs (stemming from loss in work productivity and increased absenteeism) contribute to the economic burden of migraine.

\section{What this study adds}

Patients with migraine in the United States who self-reported $\geq 4$ headache days in the previous month experienced loss in work productivity, activity impairment, and increased health care resource utilization despite taking acute and/or preventive migraine medication in comparison with propensity-matched controls free of migraine.

The burden of migraine, as measured by direct and indirect costs, is potentially a target for intervention, with wider use of effective acute and preventive treatment having the potential to reduce the burden of migraine to individuals and society.

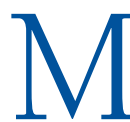
igraine is a disabling neurologic disease that affects over 1 billion people globally and is the secondleading cause of years lived with disability. ${ }^{1,2}$ In the United States, an estimated 38-40 million people have migraine, with roughly $23 \%$ of households having at least 1 family member with migraine. ${ }^{3,4}$ Migraine negatively affects daily functioning, work productivity, and health-related quality of life. ${ }^{5-10}$ The effect of migraine is often more pronounced with greater headache frequency, with 1 real-world analysis reporting greater disability and poorer health status among individuals with $\geq 4$ versus $\leq 3$ monthly headache days. ${ }^{11}$ 
Individuals with migraine are more likely to have many comorbidities, including psychiatric, cardiovascular, and cerebrovascular diseases, ${ }^{12-14}$ which may contribute to the burden and disability of migraine.

The annual cost of migraine, based on 2014 cost estimates and prevalence data from the National Health and Nutrition Examination Survey, is $\$ 78$ billion in the United States alone. ${ }^{15}$ More frequent visits to health care professionals (HCPs) and/or the emergency department (ED) and hospitalizations contribute to the higher direct costs of migraine care. ${ }^{16-18}$ Furthermore, data from the International Burden of Migraine Study (IBMS) and the American Migraine Prevalence and Prevention (AMPP) study suggest that direct and indirect costs increase with increasing frequency of migraine, 5,19,20

Guideline-recommended treatment options are available for the acute and preventive treatment of migraine pain and associated symptoms. ${ }^{21-23}$ According to a recent position paper from the American Headache Society, acute medications, including nonsteroidal anti-inflammatory drugs, acetaminophen, and analgesics (nonopioid or caffeinated combinations) may be recommended for individuals with mild to moderate migraine and migraine-specific acute medications (e.g., triptans and ergotamine derivatives) for those with moderate to severe or difficult-to-treat migraine. ${ }^{21}$ Further, individuals with $\geq 4$ headache days per month may benefit from migraine preventive therapies (e.g., traditional oral preventives, monoclonal antibodies targeting the calcitonin gene-related peptide [CGRP] or its receptor, onabotulinumtoxinA for chronic migraine only, and biobehavioral therapies) according to recent guidelines and evidence from clinical trials of CGRP pathway-targeted therapeutics. ${ }^{21,24,25}$ In addition, the approved indication for members of the most recently approved class of preventive treatments, CGRP pathway-targeted monoclonal antibodies, in Europe and several other countries designates that these medications are to be used in patients with $\geq 4$ headache days per month.

Many previous population studies have addressed the burden of migraine in highly specialized samples, such as headache center patients or employees of particular organizations. Few studies have addressed the burden of migraine in individuals who may be candidates for preventive therapy who continue to experience headache despite using acute and/or preventive prescription migraine medications. ${ }^{8}$ For this study, our goal was to assess the burden, including direct and indirect costs, of migraine in a sample of patients taking medication for migraine who might be eligible for preventive treatment based on headache frequency. We used data from the 2016 U.S. National Health and Wellness Survey (NHWS) to characterize comorbidities, work productivity and activity impairment, all-cause health care resource utilization (HRU), and associated direct and indirect costs among people who reported an HCP diagnosis of migraine and $\geq 4$ monthly headache days while currently using acute and/or preventive migraine medications.

\section{Methods}

\section{Study Design}

This was a retrospective, observational study examining data obtained from the 2016 U.S. NHWS. The 2016 NHWS was a cross-sectional internet-based questionnaire that was self-administered to a nationwide sample of U.S. adults (aged 18 years or older). Data from the Current Population Survey, conducted by the U.S. Census, were used to identify the relative proportions of age, gender, and racial/ethnic groups. These proportions were then mimicked during recruiting of panel members (using a random stratified sampling framework) to ensure that the final NHWS sample matched U.S. demographic proportions.

\section{Study Participants}

Survey respondents were recruited through an existing, general purpose (i.e., not health care specific) web-based consumer panel. All panelists explicitly agreed to be a panel member, register with the panel through a unique email address, and complete an in-depth demographic registration profile. The 2016 NHWS was granted exemption from review by the Pearl Institutional Review Board (Indianapolis, IN).

This study included respondents who reported an HCP diagnosis of migraine, completed the migraine module, and reported $\geq 4$ headache days in the previous 30 days while currently taking acute and/or preventive prescription medications for the treatment of migraine. As part of the survey, treated patients with migraine were asked to provide the medication name, which was later classified as either acute or preventive as part of the analysis. For comparison, nonmigraine respondents matched based on demographic and health history variables were also included as controls.

\section{Outcome Measures}

Sociodemographic and General Health Characteristics. The 2016 NHWS consisted of a base survey component, which captured demographics, diseases experienced and diagnosed, health outcomes, and various disease (e.g., migraine) and nondisease (e.g., vaccination) modules. All respondents were required to complete the base component, and a subset of persons with a self-reported migraine diagnosis by an HCP was randomly selected (based on a priori assigned probability) to complete the disease module for migraine, which captured disease-specific details such as age of diagnosis, severity, current treatments used and whether these treatments were acute or preventive, and other relevant validated instruments.

Once a medication was reported to be used, the respondent was instructed, "You indicated you use the following prescription medications. Please indicate if you use each medication for treatment or prevention of migraines." All data were selfreported. No medical records or other documentation were accessed. 


\section{Burden of Illness Among People with Migraine and $\geq 4$ Monthly Headache Days While Using Acute and/or Preventive Prescription Medications for Migraine}

General sociodemographic variables (e.g., age, gender, race/ ethnicity, marital status, education, household income, and health insurance type) and disability status were captured as part of the NHWS. The NHWS also captured body mass index (BMI), current and previous smoking status, alcohol use, and exercise behavior. Survey respondents were asked to report any comorbid conditions, including those prevalent among patients with migraine (e.g., depression, generalized anxiety, and insomnia). The Charlson Comorbidity Index (CCI) score was also calculated and weighed the presence of the following conditions and summed the result: HIV/AIDS, metastatic tumor, lymphoma, leukemia, any tumor, moderate/severe renal disease, hemiplegia, diabetes, mild liver disease, ulcer disease, connective tissue disease, chronic pulmonary disease, dementia, cerebrovascular disease, peripheral vascular disease, myocardial infarction, congestive heart failure, and diabetes with end organ damage. ${ }^{26} \mathrm{~A}$ greater CCI score indicates greater comorbid burden on the patient.

Work Productivity and Activity Impairment. Impairment in work and daily activities was measured by the Work Productivity and Activity Impairment questionnaire, a 6-item validated instrument that consists of metrics for absenteeism (percentage of work time missed because of one's health in the previous 7 days), presenteeism (percentage of impairment or reduced productivity experienced while at work in the previous 7 days because of one's health), overall work productivity loss (estimated based on combination of presenteeism and absenteeism), and activity impairment (percentage of impairment in daily activities because of one's health in the previous 7 days). ${ }^{27}$ Data for activity impairment were captured for all respondents; however, only respondents who reported being full-time or part-time employed provided data for absenteeism, presenteeism, and overall work impairment.

All-Cause Health Care Resource Utilization. All-cause HRU, defined as self-reported HCP visits, ED visits, or hospitalizations in the previous 6 months, was measured. The phrasing was intentionally kept vague to ensure that all medical conditions were included.

Estimated Annualized Direct and Indirect Costs. For each respondent, all-cause direct cost of care was estimated by summing the costs of each type of visit (e.g., HCP visit, ED visit, and hospitalization) and multiplying the projected number of annual visits by the average cost of the visit. The annual number of visits was projected by doubling the number of visits reported in the previous 6 months. Similar methods for estimation of annualized direct costs (using 3 months of data multiplied by 4) have been used in previous cost analyses for migraine. ${ }^{28,29}$ Average costs of HCP and ED visits and hospitalization were obtained from the 2014 Medical Expenditure Panel Survey data. Medication-related direct costs were not included in the analysis.
For each employed respondent, indirect costs were derived from the median weekly income figures from the 2014 U.S. Bureau of Labor Statistics. For each of these respondents, an hourly rate was estimated, based on the median weekly income divided by the duration of the typical work week. The number of hours missed per week due to absenteeism and presenteeism was multiplied by the hourly rate to determine the total lost wages per week, which were used to calculate annual lost wage estimates for each employed respondent. This approach, the human capital approach, has been widely used to estimate indirect costs. ${ }^{30,31}$

\section{Statistical Analysis}

A 1:1 matched control group of respondents without migraine was created using a propensity score-matching procedure. Respondents with and without migraine were compared with respect to demographics and health history variables using chi-square tests and one-way analysis of variance (ANOVA). Variables that differed between groups at a level of $P<0.25$ were then entered in a logistic regression model to predict migraine presence (i.e., migraine vs. no migraine). Variables for the propensity match included age, gender, CCI score, income, education, insurance, BMI, and smoking status. Because of the small sample sizes, the number of variables entered in the logistic regression was restricted to minimize the total number of variables so that the model would converge.

Propensity score values from this model were saved and used as the basis for matching each respondent with migraine with a respondent without migraine whose propensity score value was identical. Specifically, a greedy-matching algorithm, which identified controls to match to a single case at up to 8 decimal places of the propensity score (and as little as 1 decimal place if no other suitable control was identified), was used. ${ }^{32}$ Matched respondents without migraine were considered a matched control. Patients with migraine treated after matching and their matched nonmigraine controls were compared with respect to demographics and health history variables using chi-square tests and one-way ANOVAs to test the degree of balance between groups and determine whether any variables should be further controlled.

Descriptive statistics (means, standard deviations for continuous variables and frequencies, and percentages for categorical variables) were reported for all measures. Patients with migraine were compared with matched nonmigraine controls using one-way ANOVA for continuous outcomes and chi-square tests for categorical outcomes.

Two-sided $P$ values of less than 0.05 were considered statistically significant. Analyses were conducted using IBM SPSS Statistics for Windows, version 23.0 (Armonk, NY). 


\section{Burden of Illness Among People with Migraine and $\geq 4$ Monthly Headache Days While Using Acute and/or Preventive Prescription Medications for Migraine}

\begin{tabular}{|c|c|c|c|}
\hline & $\begin{array}{l}\text { Treated Patients } \\
\text { with Migraine } \\
(\mathrm{n}=197)\end{array}$ & $\begin{array}{c}\text { Nonmigraine } \\
\text { Controls } \\
(\mathrm{n}=197)\end{array}$ & $P$ Value \\
\hline Age, mean (SD), years & $40.7 \quad(13.2)$ & $40.2 \quad(14.0)$ & 0.719 \\
\hline \multicolumn{4}{|l|}{$\begin{array}{l}\text { Age, mean (SD), years } \\
\text { CCI }\end{array}$} \\
\hline Score, mean (SD) & $0.72 \quad(1.5)$ & $(1.2)$ & 0.428 \\
\hline \multicolumn{4}{|l|}{ Categories, ${ }^{\mathrm{a}} \mathrm{n}(\%)$} \\
\hline CCI: 0 & $133(67.5)$ & $137 \quad(69.5)$ & 0.794 \\
\hline CCI: 1 & $28 \quad(14.2)$ & $31 \quad(15.7)$ & - \\
\hline CCI: 2 & $21 \quad(10.7)$ & $16 \quad(8.1)$ & - \\
\hline CCI: $3+$ & $15 \quad(7.6)$ & $13 \quad(6.6)$ & - \\
\hline Women, n (\%) & $164(83.2)$ & $162(82.2)$ & 0.790 \\
\hline \multicolumn{4}{|c|}{ Annual household income ${ }^{\mathrm{a}} \mathrm{n}(\%)$} \\
\hline$<\$ 25,000$ & $36 \quad(18.3)$ & $37(18.8)$ & 0.989 \\
\hline$\$ 25,000-\$ 50,000$ & $60 \quad(30.5)$ & $59 \quad(29.9)$ & - \\
\hline$\$ 50,000-\$ 75,000$ & $31 \quad(15.7)$ & $30(15.2)$ & - \\
\hline$\geq \$ 75,000$ & $59 \quad(29.9)$ & $62 \quad(31.5)$ & - \\
\hline Declined to answer & $11 \quad(5.6)$ & $9 \quad(4.6)$ & - \\
\hline $\begin{array}{l}\text { 4-year college degree or } \\
\text { higher, } n(\%)\end{array}$ & $74 \quad(37.6)$ & $75 \quad(38.1)$ & 0.917 \\
\hline $\begin{array}{l}\text { Currently has health } \\
\text { insurance, } \mathrm{n}(\%)\end{array}$ & $184(93.4)$ & $190(96.4)$ & 0.168 \\
\hline \multicolumn{4}{|l|}{ BMI category, ${ }^{a}$ n (\%) } \\
\hline Underweight & $7 \quad(3.6)$ & $5 \quad(2.5)$ & 0.937 \\
\hline Normal weight & $62 \quad(31.5)$ & $57(28.9)$ & - \\
\hline Overweight & $59 \quad(29.9)$ & $60 \quad(30.5)$ & - \\
\hline Obese & $67(34.0)$ & $73 \quad(37.1)$ & - \\
\hline Unknown & $2 \quad(1.0)$ & $2 \quad(1.0)$ & - \\
\hline \multicolumn{4}{|l|}{ Smoking status, ${ }^{\text {a }}$ n (\%) } \\
\hline Current smoker & $42 \quad(21.3)$ & $47 \quad(23.9)$ & 0.721 \\
\hline Former smoker & $52(26.4)$ & $46 \quad(23.4)$ & - \\
\hline Never smoker & $103 \quad(52.3)$ & $104(52.8)$ & - \\
\hline \multicolumn{4}{|c|}{ Self-reported comorbidities, n (\%) } \\
\hline Depression & $115 \quad(58.4)$ & $55 \quad(27.9)$ & $<0.001$ \\
\hline $\begin{array}{l}\text { Generalized anxiety } \\
\text { disorder }\end{array}$ & $30(15.2)$ & $17 \quad(8.6)$ & 0.043 \\
\hline Sleep apnea & $19 \quad(9.6)$ & $(6.6)$ & 0.268 \\
\hline \multicolumn{4}{|c|}{ Occupational disability status, ${ }^{a}$ n (\%) } \\
\hline Not disabled & $166 \quad(84.3)$ & $186 \quad(94.4)$ & 0.003 \\
\hline Short-term disability & $4 \quad(2.0)$ & $0 \quad(0.0)$ & - \\
\hline Long-term disability & $27(13.7)$ & $11 \quad(5.6)$ & - \\
\hline \multicolumn{4}{|c|}{$\begin{array}{l}\text { aP values represent omnibus chi-square test results. } \\
B M I=\text { body mass index; } C C I=\text { Charlson Comorbidity Index; } N S=\text { not significant; } \\
S D=\text { standard deviation. }\end{array}$} \\
\hline
\end{tabular}

\section{Results}

\section{Patient Demographics and Characteristics}

Of the 97,503 patients who participated in the 2016 NHWS, 9,579 self-reported a diagnosis of migraine by an HCP, and 933 were selected to complete the migraine module (Supplementary Figure 1, available in online article). Analysis included 197 respondents who reported $\geq 4$ headache days in the previous month while taking acute and/or preventive medications for migraine (referred to as treated patients with migraine) and a matched control group of 197 respondents who did not self-report a diagnosis of migraine.

Acute medications commonly used for management of migraine among the treated respondents included prescription and over-the-counter (OTC) medications, including sumatrip$\tan$ (58 patients, 29.4\%); a combination of acetaminophen, aspirin, and caffeine (45 patients, 22.8\%); ibuprofen (42 patients, $21.3 \%$ ); and naproxen (25 patients, $12.7 \%$ ). Preventive medications included topiramate (22 patients, 11.2\%); amitriptyline (11 patients, 5.6\%); and onabotulinumtoxinA (11 patients, $5.6 \%$ ). More than half reported using OTC medications or herbal products. A complete listing, including patient-reported reason for medication use (treatment vs. preventive), is provided in Supplementary Table 1 (available in online article).

The treated migraine and nonmigraine groups were sociodemographically balanced (Table 1). Most respondents were women, with a mean age of 40 years. Compared with nonmigraine controls, a greater proportion of the treated migraine group reported being on long-term disability (13.7\% vs. 5.6\%), being diagnosed with depression $(58.4 \%$ vs. $27.9 \%, P<0.001)$, or being diagnosed with generalized anxiety disorder (15.2\% vs. $8.6 \%, P=0.043)$. Based on mean $C C I$ scores, the overall comorbidity burden among treated respondents with migraine was numerically greater than nonmigraine matched controls but not statistically significant.

\section{Work Productivity and Activity}

Among employed respondents, absenteeism (11.8\% vs. 6.3\%, $P=0.030$ ), presenteeism (36.0\% vs. $17.5 \%, P<0.001)$, and overall work impairment $(41.0 \%$ vs. $20.9 \%, P<0.001)$ were significantly higher in treated migraine patients versus nonmigraine controls (Table 2). Relative to matched controls, treated migraine patients reported greater activity impairment due to health reasons ( $45.4 \%$ vs. $25.4 \%, P<0.001$; Figure 1 ).

\section{Health Care Resource Utilization}

All-cause HRU during the previous 6 months was higher among treated migraine patients than the nonmigraine matched controls (Table 2). Relative to nonmigraine controls, treated migraine patients reported significantly more overall HCP visits (7.55 vs. $4.43, P<0.001)$ and $E D$ visits ( 0.48 vs. $0.25, P=0.030)$, whereas the number of hospitalizations $(0.19$ vs. $0.16, P=0.610$ ) was greater but not statistically significant (Figure 2). Among HCP visits, the numbers of general practitioner visits ( 2.58 vs. $2.12, P=0.146)$ and neurologist visits (2.09 vs. $2.00, P=0.924)$ were greater for treated patients with migraine compared with nonmigraine controls, but differences were not statistically significant (Figure 2). 


\section{Burden of Illness Among People with Migraine and $\geq 4$ Monthly Headache Days \\ While Using Acute and/or Preventive Prescription Medications for Migraine}

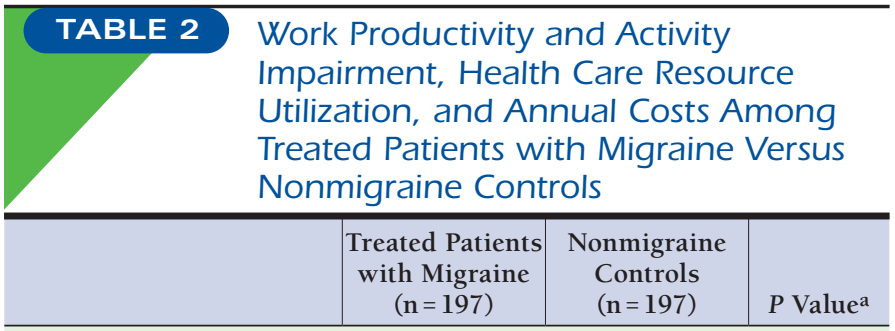

Work productivity and activity impairment in the previous 7 days, mean (SD), \%

\begin{tabular}{l|rr|rr|r}
\hline Absenteeism & 11.8 & $(20.7)$ & 6.3 & $(17.8)$ & 0.030 \\
\hline Presenteeism & 36.0 & $(31.5)$ & 17.5 & $(25.5)$ & $<0.001$ \\
\hline Overall work impairment & 41.0 & $(33.9)$ & 20.9 & $(29.2)$ & $<0.001$ \\
\hline Activity impairment & 45.4 & $(30.2)$ & 25.4 & $(29.2)$ & $<0.001$ \\
\hline
\end{tabular}

All-cause health care resource utilization during the previous 6 months, mean (SD)

\begin{tabular}{l|rr|rr|r}
\hline HCP visits $^{b}$ & 7.6 & $(9.6)$ & 4.4 & $(7.7)$ & $<0.001$ \\
\hline GP visits & 2.6 & $(2.5)$ & 2.1 & $(2.3)$ & 0.146 \\
\hline Neurologist visits & 2.1 & $(1.8)$ & 2.0 & $(2.0)$ & 0.924 \\
\hline ED visits & 0.5 & $(1.2)$ & 0.3 & $(0.9)$ & 0.030 \\
\hline Hospitalizations & 0.2 & $(0.6)$ & 0.2 & $(0.8)$ & 0.610 \\
\hline
\end{tabular}

Estimated annual costs, mean (SD), USD

\begin{tabular}{l|rr|rrr}
\hline Total all-cause direct costs & 24,500 & $(38,512)$ & 15,319 & $(34,532)$ & 0.013 \\
\hline HCP visits & 15,967 & $(20,786)$ & 9,630 & $(15,486)$ & $<0.001$ \\
\hline Hospitalizations & 6,849 & $(21,741)$ & 4,789 & $(21,893)$ & 0.349 \\
\hline ED visits & 1,684 & $(4,160)$ & 900 & $(3,129)$ & 0.035 \\
\hline Total indirect costs & 14,771 & $(14,732)$ & 5,765 & $(9,159)$ & $<0.001$ \\
\hline Absenteeism & 4,134 & $(8,035)$ & 1,537 & $(5,078)$ & 0.003 \\
\hline Presenteeism & 10,637 & $(11,781)$ & 4,228 & $(7,195)$ & $<0.001$ \\
\hline
\end{tabular}

aP values represent chi-square test results.

${ }^{b}$ The number of HCP visits refers to visits to all HCPs (including GPS and neurologists).

$E D=$ emergency department; $G P=$ general practitioner; $H C P=$ health care professional; $S D=$ standard deviation; $U S D=U . S$. dollars.

\section{Annualized Direct and Indirect Cost}

Estimated annualized mean all-cause direct cost due to HRU was significantly greater among treated migraine patients than nonmigraine matched controls ( $\$ 24,499.90$ vs. $\$ 15,318.91$, $P=0.013$; Table 2 and Figure 3). The costs of HCP visits $(\$ 15,967.09$ vs. $\$ 9,630.44, P<0.001)$ and $E D$ visits $(\$ 1,683.78$ vs. $\$ 899.66, P=0.035)$ were significantly greater for treated patients with migraine than for nonmigraine controls.

Treated migraine patients incurred significantly higher estimated mean overall annualized indirect costs than nonmigraine controls ( $\$ 14,770.57$ vs. $\$ 5,764.93, P<0.001$; Figure 3 ). Absenteeism and presenteeism contributed to the overall difference, since the costs of each were significantly greater for treated patients with migraine than for nonmigraine controls (absenteeism: $\$ 4,133.68$ vs. $\$ 1,536.51, P=0.003$; presenteeism: $\$ 10,636.89$ vs. $\$ 4,228.41, P<0.001)$.

\section{Discussion}

The results from this study further expand the current knowledge of the burden of migraine among treated patients reporting $\geq 4$ headache days in the preceding month while using acute and/or preventive prescription migraine medication. Despite taking migraine medication, this migraine group was more likely to have reduced work productivity, increased activity impairment, and increased all-cause HRU, which contributed to higher direct and indirect costs versus the matched nonmigraine control.

Reduced productivity and activity limitations in the migraine group may have been a direct consequence of migraine, although comorbidities may also have contributed. The migraine group had higher comorbidity scores and an elevated relative frequency of depression and anxiety. These findings are consistent with previous studies, including the Chronic Migraine Epidemiology and Outcomes (CaMEO) study and the Migraine in America Symptoms and Treatment (MAST) study. 12,33-36 Comorbid conditions may complicate migraine diagnosis and treatment, worsen quality of life, increase migraine-related disability, and increase the risk of progression from episodic to chronic migraine..$^{12,37,38}$ This, in turn, often increases direct and indirect costs for the treatment of migraine. ${ }^{16,39}$ An analysis of Thomson Medstat MarketScan Commercial Claims and Encounters and Health and Productivity Management databases from 1999 to 2000 found the cost of outpatient care in patients with migraine (e.g., medication costs and HCP visits) to be significantly greater with comorbid depression and/or anxiety. ${ }^{39}$

Migraine patients in the United States who were treated with OTC and/or prescription medications had a 1.8-fold increase in activity impairment relative to nonmigraine controls. Employed, treated patients with migraine had a 1.9-fold increase in absenteeism, a 2.1-fold increase in presenteeism, and a 2 -fold increase in overall work productivity loss. Furthermore, patients with migraine using prescription migraine medication reported significantly higher all-cause HRU, driven primarily by significantly more visits to HCPs and the ED relative to controls. These data are in line with previous studies reporting reduced work productivity and increased HRU in patients with migraine. ${ }^{7,40}$

A recent retrospective analysis of NHWS data for European patients with migraine (self-reported physician diagnosis of migraine and $\geq 4$ monthly headache days) found a 1.7 -fold increase in presenteeism, 1.5-fold increase in absenteeism, 1.7-fold increase in work productivity impairment, and 1.6-fold increase in activity impairment compared with nonmigraine controls. ${ }^{8}$ In addition, European patients with migraine reported more HCP or ED visits and more hospitalizations than did nonmigraine controls. ${ }^{8}$ The current results from a U.S. sample from the NHWS dataset support the findings of that previous analysis in European migraine patients, ${ }^{8}$ showing a 


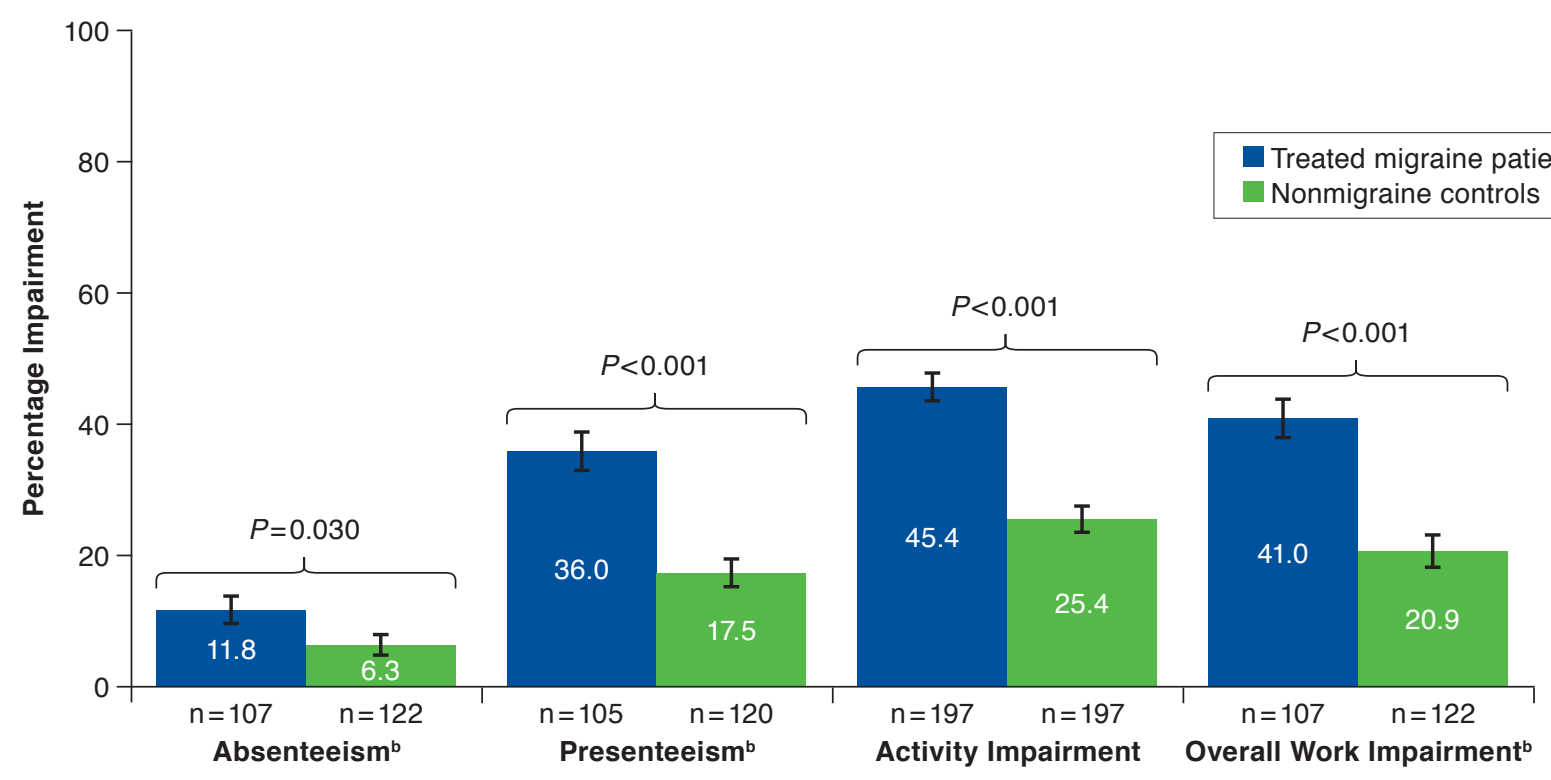

Note: Error bars represent standard error. P values represent omnibus ANOVA results.

a Measured using the Work Productivity and Activity Impairment questionnaire.

${ }^{b}$ Measured only for employed respondents.

ANOVA = analysis of variance.

substantial burden of migraine in patients who are experiencing $\geq 4$ monthly headache days, with or without treatment.

The findings from this study are also consistent with those from the My Migraine Voice Survey, which found that adults with migraine who reported $\geq 4$ monthly migraine days in the 3 months preceding survey administration, with prespecified criteria of $90 \%$ having used preventive migraine treatment ( $80 \%$ with history of $\geq 1$ treatment failure), had a high rate of ED visits (38\%) and overnight stays in the hospital (23\%) due to migraine. ${ }^{10}$ Furthermore, HRU was greater among the subset of individuals who had failed to respond to $\geq 2$ migraine preventive therapies. ${ }^{10,41}$

Although HCP visits overall were significantly greater for migraine patients than for nonmigraine controls, the number of general practitioner and neurologist visits did not differ significantly between migraine patients and controls. These results are in keeping with a previous study showing that specialty care visits accounted for only approximately $27 \%$ of visits for migraine, with primary care visits accounting for the majority of visits. ${ }^{42}$ The lack of difference in neurologist visits between migraine patients and controls is of note and suggests that patients may not be receiving adequate specialty care or follow-up or that other, nonmigraine neurological conditions in the control cohort may have driven the number of neurologist office visits among controls. Further studies would be required to confirm the reason that neurologist visits did not differ significantly for migraine patients and nonmigraine controls.

Treated migraine patients in this study reported significantly greater all-cause direct ( $\$ 24,500$ vs. $\$ 15,319$ for nonmigraine controls) and indirect costs $(\$ 14,771$ vs. $\$ 5,765$ for nonmigraine controls). Greater HRU among treated migraine patients in this study may have contributed to a difference of $\$ 9,181$ in all-cause direct costs. Estimated annualized indirect costs for treated migraine patients were $\$ 9,006$ higher (2.6-fold greater) than the nonmigraine controls, due to reduced work productivity and higher absenteeism. Costs may be even greater than estimated here, since this study did not account for costs associated with increased absenteeism and/or loss in work productivity resulting from individuals who retired early, were occupationally disabled, or left careers.

These findings are consistent with published studies characterizing the economic costs of migraine in the general and key subpopulations (e.g., chronic migraine vs. episodic migraine). ${ }^{5,13,14,16,19,20,40,43-44} \mathrm{~A}$ study by Hawkins et al. (2007) analyzing data from Thomson Medstat's Health and Productivity Management database for 2002 to 2003 found that patients with migraine had a significantly higher total indirect cost than matched nonmigraine controls ( $\$ 4,453$ vs. $\$ 1,619$ per year, respectively), resulting from increased absenteeism, short-term disability, and workers' compensation. ${ }^{44}$ A separate analysis of 


\section{FIGURE 2 All-Cause Health Care Resource Utilization During the Previous 6 Months}

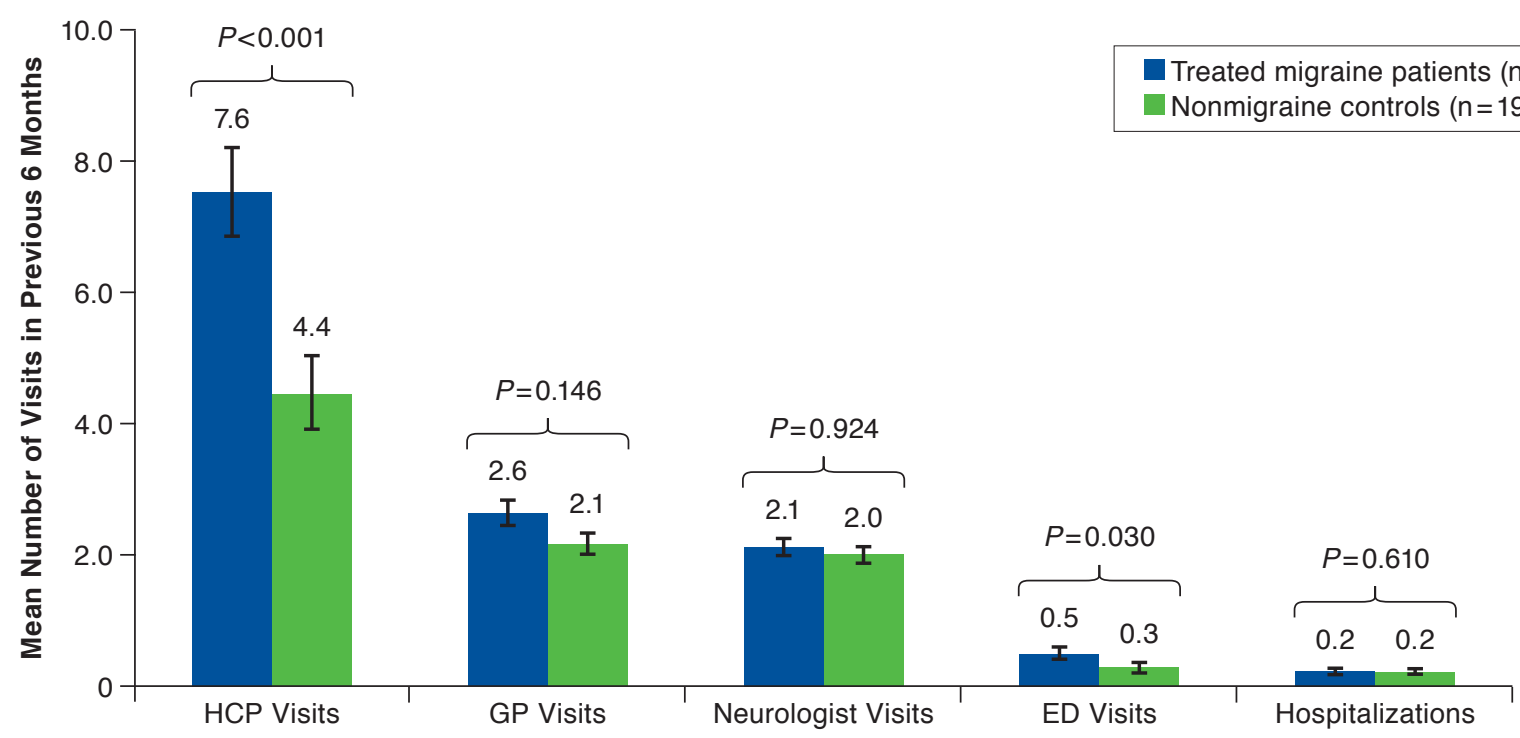

Note: Error bars represent standard error. $P$ values represent omnibus ANOVA results.

ANOVA = analysis of variance; $E D=$ emergency department; $G P=$ general practitioner; $H C P=$ health care professional.

the 2004 data from Thomson Medstat's Commercial Claims and Encounters database reported that average health care expenditures for patients with migraine were $\$ 2,571$ higher compared with matched controls ( $\$ 7,007$ vs. $\$ 4,436$ per person per year). ${ }^{43}$ Our results support the findings of these previous analyses.

A cost study conducted as part of the Eurolight project (a cross-sectional survey conducted across 8 European countries from 2008 to 2009) reported a mean per-person annual cost of migraine of $€ 1,222 .{ }^{28}$ Notably, the study found that outpatient care and work productivity were key contributors to high direct and indirect costs. ${ }^{28}$ We observed similar results, with absenteeism and presenteeism driving costs.

Furthermore, the AMPP and IBMS studies reported that direct and indirect costs increase substantially as headache frequency increases. ${ }^{5,19,20} \mathrm{~A}$ recent retrospective analysis of Truven Health MarketScan Research Databases from 2008 to 2013 estimated total costs for migraine patients ( $N=71,742)$, including those on acute and/or preventive migraine medication, to be $\$ 8,924$ (in 2014 U.S. dollars) greater than those for matched nonmigraine controls. ${ }^{14}$ Further, direct all-cause health care costs were $\$ 6,575$ higher among migraine patients. ${ }^{14}$ Mean annualized indirect cost based on work days lost due to disability (short- and long-term) and workplace absence was $\$ 2,350$ higher among migraine patients. ${ }^{14}$ Collectively, these studies show a significant economic burden for individuals with migraine.
Differences in cost estimates reported here and in other previously reported studies may be attributed to study population and design. This study focused on a highly select subset of the general migraine population: individuals with HCPdiagnosed migraine who had experienced $\geq 4$ headache days in the preceding month despite taking acute and/or preventive prescription migraine medications. These patients with more difficult-to-treat migraine may have increased HRU due to persistent burden despite prescription medications, and this may explain why direct costs for these patients were higher than indirect costs, while other studies have demonstrated higher indirect costs than direct costs for people with migraine. Further, the study design may influence cost estimates, since both data sources (e.g., web-based survey and claims databases) and the information captured and processed can vary greatly between studies.

Taken together, these data show a pervasive negative effect of migraine on many important aspects of life and highlight a significant unmet need in the management and treatment of migraine as of 2016. In this study, patients with migraine who self-reported $\geq 4$ headache days continued to experience loss in work productivity, activity impairment, and increased HRU despite using acute and/or preventive prescription migraine medication. This suggests that the poor management of migraine in patients on active treatment may be attributed to the limited availability of effective and well-tolerated pharmacologic treatment options at the time this study was conducted. The recent approval of a new class of preventive pharmacologic 


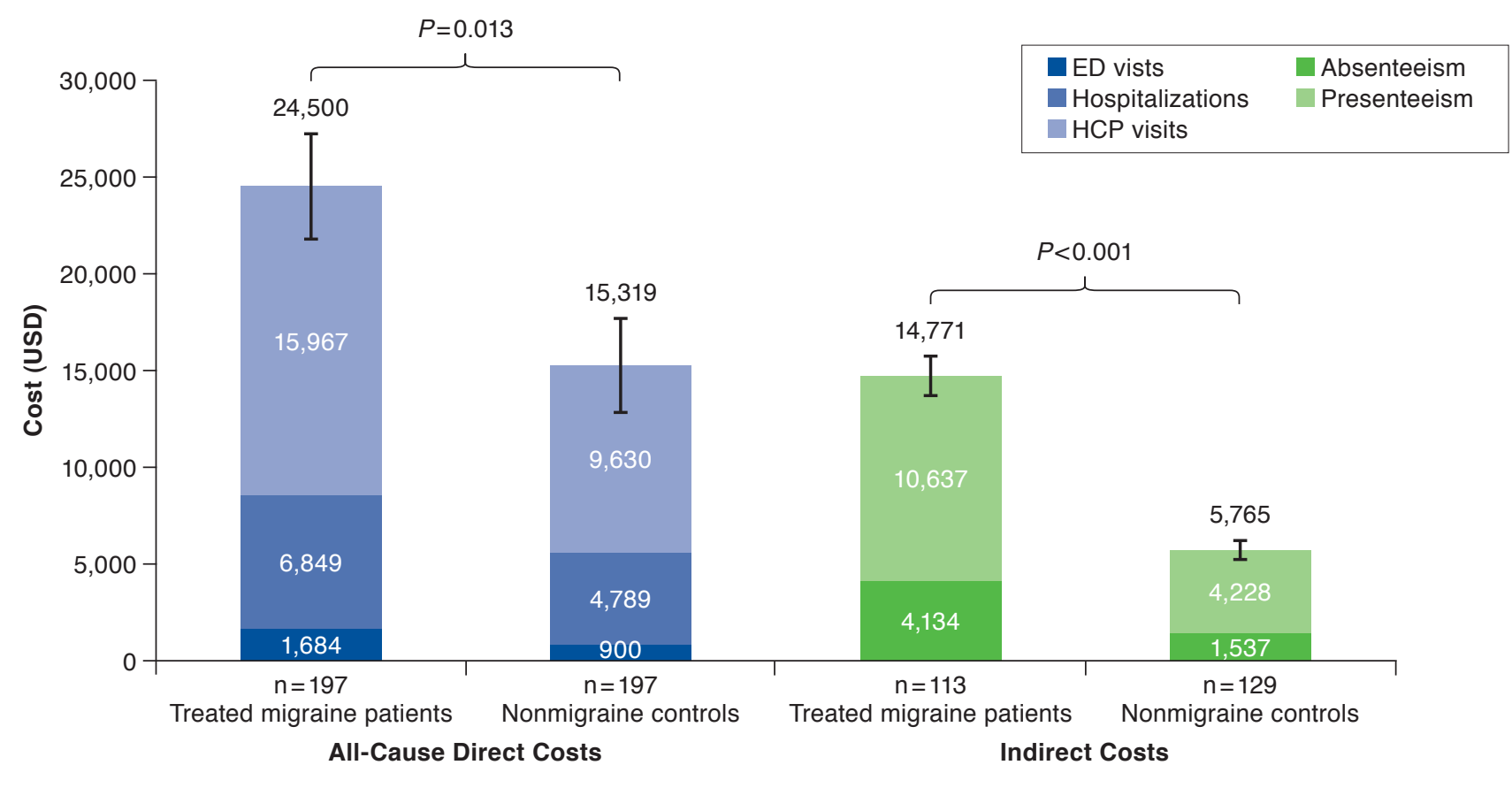

Note: Error bars represent standard error. $P$ values represent omnibus ANOVA results.

ANOVA = analysis of variance; $E D=$ emergency department; $H C P=$ health care professional; $U S D=U . S$. dollars.

treatments developed to specifically target the pathophysiology of migraine, monoclonal antibodies that antagonize CGRP or its receptor, may help to meet this unmet need coupled with a greater use of other effective modalities, including empirically supported biobehavioral therapies and neurostimulation for prevention. ${ }^{45-49}$

\section{Limitations}

This study has limitations, including the retrospective, crosssectional study design. The data captured by the survey were patient reported and cannot be independently verified by an HCP or medical record. Inaccurate recall, misunderstandings, and false reporting may introduce potential bias and limit the interpretation of the data. This is likely evidenced in the way that respondents were asked whether each medication that they endorsed using was used for the "treatment or prevention" of migraine. We have interpreted "treatment" as acute and "prevention" as preventive therapies; however, patients' potential misunderstandings were evident in how some medications were classified.

Although the NHWS is broadly representative of the adult population, individuals without access to or comfort with online surveys may be underrepresented. In addition, our sample of individuals with HCP-diagnosed migraine who reported $\geq 4$ headache days in the preceding month despite taking acute and/or preventive migraine medications is a selected sample of treated individuals with active, diagnosed migraine and is thus representative of a treated sample of patients with migraine but not of the general migraine population.

Although individuals were matched based on propensity score across different variables (e.g., age, gender, and BMI), variables that were not considered in this analysis may introduce differences in patient-reported outcomes. Annualized numbers of HCP visits, ED visits, and hospitalizations, as well as direct and indirect costs, were estimated based on 6-month data. This study also did not account for the costs associated with medication overuse headache and detoxification or costs associated with increased absenteeism and/or loss in work productivity resulting from individuals who retired early, were occupationally disabled, or left careers.

Medication adherence was not assessed and thus was not accounted for in this analysis, and patients who were adherent to their acute or preventive medications may have experienced better outcomes than those who were not. In addition, the presence of comorbidities in this patient population may have contributed to direct and indirect costs, confounding the ability to distinguish the cost of migraine from the cost of comorbid conditions. This analysis also did not account for the effect of behavioral and nonpharmacologic therapeutic options. 


\section{Burden of Illness Among People with Migraine and $\geq 4$ Monthly Headache Days While Using Acute and/or Preventive Prescription Medications for Migraine}

\section{Conclusions}

Data from this study support and expand on the findings of previous studies, ${ }^{8,10}$ showing that the overall burden of migraine, as measured by presence of comorbidities, rates of long-term disability, loss in work productivity, increased all-cause HRU, and increased direct and indirect costs, is substantial among migraine patients who self-reported $\geq 4$ headache days in the previous month despite using acute and/or preventive prescription migraine medication. Moreover, this emphasizes the need for effective management of migraine, including the appropriate use of new pharmacologic and established multidisciplinary migraine preventive treatments to lessen the effect and burden of migraine.

\section{Authors}

DAWN C. BUSE, PhD, Albert Einstein College of Medicine, Bronx, New York; MARIANNA S. YUGRAKH, MD, Department of Neurology, Columbia University Medical Center, New York, New York; and LULU K. LEE, PhD, Kantar, San Mateo, California. JVAWNNA BELL, MPH, MBA, and JOSHUA M. COHEN, MD, MPH, FAHS, Teva Branded Pharmaceutical Products RED, West Chester, Pennsylvania. RICHARD B. LIPTON, MD, Albert Einstein College of Medicine and Montefiore Medical Center, Bronx, New York.

AUTHOR CORRESPONDENCE: Dawn C. Buse, PhD, Department of Neurology, Albert Einstein College of Medicine, 1250 Waters Pl., Bronx, NY 10461. Tel.: 858.203.7691; Email: dbuse@montefiore.org.

\section{DISCLOSURES}

This study was funded by Teva Pharmaceutical Industries (Petach Tikva, Israel). Cohen is an employee of Teva Branded Pharmaceutical Products R\&D (USA); Bell was an employee of Teva Pharmaceutical Industries at the time of this study and holds stock/stock options in Teva Pharmaceutical Industries. Lee is an employee of Kantar, which received funding from Teva Pharmaceutical Industries for data analyses performed for this study. Buse has served as a paid consultant to Amgen/Novartis, Allergan, Biohaven, Eli Lilly, Promius/Dr. Reddy's, and Teva Pharmaceuticals, but she was not compensated financially for work on this study. Yugrakh has received research support from Teva Pharmaceuticals and Cefaly Technology. Lipton has received research support from the NIH, the Migraine Research Foundation, and the National Headache Foundation; holds stock options in eNeura Therapeutics and Biohaven Holdings; serves as consultant, advisory board member, or has received honoraria from the American Academy of Neurology, Alder, Allergan, the American Headache Society, Amgen, Autonomic Technologies, Avanir, Biohaven, BioVision, Boston Scientific, Dr. Reddy's, electroCore, Eli Lilly, eNeura Therapeutics, GlaxoSmithKline, Merck, Pernix, Pfizer, Supernus, Teva, Trigemina, Vector, and Vedanta.

This study was presented as a poster at the American Academy of Neurology 2018 Annual Meeting, April 21-27, 2018, in Los Angeles, CA; PAINWeek 2018, September 4-8, 2018, in Las Vegas, NV; and the 2017 European Headache Federation (EHF) Congress, December 1-3, 2017, in Rome, Italy.

\section{ACKNOWLEDGMENTS}

The authors thank Disha Patel, PhD, and Lindsay Tannenholz, PhD (Chameleon Communications International with funding from Teva Pharmaceutical Industries) for editorial assistance in the preparation of this report.

\section{REFERENCES}

1. Institute for Health Metrics and Evaluation. Global Burden of Diseases, Injuries, and Risk Factors Study (GBD). 2018. Available at: http://vizhub. healthdata.org/gbd-compare. Accessed June 16, 2020.

2. GBD 2017 Disease and Injury Incidence and Prevalence Collaborators. Global, regional, and national incidence, prevalence, and years lived with disability for 354 diseases and injuries for 195 countries and territories, 1990-2017: a systematic analysis for the Global Burden of Disease Study 2017. Lancet. 2018;392(10159):1789-858.

3. Bonafede M, Cai Q, Cappell K, et al. Factors associated with direct health care costs among patients with migraine. J Manag Care Spec Pharm 2017;23(11):1169-76. Available at: https://www.jmcp.org/doi/10.18553/ jmcp. 2017.23.11.1169.

4. Lipton RB, Stewart WF, Diamond S, Diamond ML, Reed M. Prevalence and burden of migraine in the United States: data from the American Migraine Study II. Headache. 2001;41(7):646-57.

5. Munakata J, Hazard E, Serrano D, et al. Economic burden of transformed migraine: results from the American Migraine Prevalence and Prevention (AMPP) Study. Headache. 2009;49(4):498-508.

6. Mahmoudzadeh Zarandi F, Raiesifar A, Ebadi A. The effect of Orem's self-care model on quality of life in patients with migraine: a randomized clinical trial. Acta Med Iran. 2016;54(3):159-64.

7. Lipton RB, Bigal ME. Migraine: epidemiology, impact, and risk factors for progression. Headache. 2005;45(Suppl 1):S3-S13.

8. Vo P, Fang J, Bilitou A, Laflamme AK, Gupta S. Patients' perspective on the burden of migraine in Europe: a cross-sectional analysis of survey data in France, Germany, Italy, Spain, and the United Kingdom. J Headache Pain 2018;19(1):82

9. Buse DC, Fanning KM, Reed ML, et al. Life with migraine: effects on relationships, career, and finances from the Chronic Migraine Epidemiology and Outcomes (CaMEO) study. Headache. 2019;59(8):1286-99.

10. Martelletti P, Schwedt TJ, Lanteri-Minet M, et al. My Migraine Voice survey: a global study of disease burden among individuals with migraine for whom preventive treatments have failed. J Headache Pain. 2018;19(1):115.

11. Ford JH, Jackson J, Milligan G, Cotton S, Ahl J, Aurora SK. A real-world analysis of migraine: a cross-sectional study of disease burden and treatment patterns. Headache. 2017;57(10):1532-44.

12. Minen MT, De Dhaem OB, Van Diest AK, et al. Migraine and its psychiatric comorbidities. 2016;87(7):741-49.

13. Bigal ME, Lipton RB. The epidemiology, burden, and comorbidities of migraine. Neurol Clin. 2009;27(2):321-34.

14. Bonafede M, Sapra S, Shah N, Tepper S, Cappell K, Desai P. Direct and indirect healthcare resource utilization and costs among migraine patients in the United States. Headache. 2018;58(5):700-14.

15. Gooch CL, Pracht E, Borenstein AR. The burden of neurological disease in the United States: a summary report and call to action. Ann Neurol. 2017;81(4):479-84.

16. Hazard E, Munakata J, Bigal ME, Rupnow MF, Lipton RB. The burden of migraine in the United States: current and emerging perspectives on disease management and economic analysis. Value Health. 2009;12(1):55-64.

17. Ford JH, Schroeder K, Nyhuis AW, Foster SA, Aurora SK. Cycling through migraine preventive treatments: implications for all-cause total direct costs and disease-specific costs. J Manag Care Spec Pharm. 2019;25(1):46-59. Available at: https://www.jmcp.org/doi/10.18553/ jmcp.2018.18058.

18. Lipton RB, Scher AI, Kolodner K, Liberman J, Steiner TJ, Stewart WF Migraine in the United States: epidemiology and patterns of health care use. Neurology. 2002;58(6):885-94. 


\section{Burden of Illness Among People with Migraine and $\geq 4$ Monthly Headache Days While Using Acute and/or Preventive Prescription Medications for Migraine}

19. Stokes M, Becker WJ, Lipton RB, et al. Cost of health care among patients with chronic and episodic migraine in Canada and the USA: results from the International Burden of Migraine Study (IBMS). Headache. 2011;51(7):1058-77.

20. Messali A, Sanderson JC, Blumenfeld AM, et al. Direct and indirect costs of chronic and episodic migraine in the United States: a web-based survey. Headache. 2016;56(2):306-22.

21. American Headache Society. The American Headache Society position statement on integrating new migraine treatments into clinical practice. Headache. 2019;59(1):1-18.

22. Silberstein SD, Holland S, Freitag F, et al. Evidence-based guideline update: pharmacologic treatment for episodic migraine prevention in adults: report of the Quality Standards Subcommittee of the American Academy of Neurology and the American Headache Society. Neurology. 2012;78(17):1337-45.

23. Loder E, Burch R, Rizzoli P. The 2012 AHS/AAN guidelines for prevention of episodic migraine: a summary and comparison with other recent clinical practice guidelines. Headache. 2012;52(6):930-45.

24. Sacco S, Bendtsen L, Ashina M, et al. European Headache Federation guideline on the use of monoclonal antibodies acting on the calcitonin gene related peptide or its receptor for migraine prevention.J Headache Pain. 2019;20(1):6.

25. Deng H, Li GG, Nie H, et al. Efficacy and safety of calcitonin-generelated peptide binding monoclonal antibodies for the preventive treatment of episodic migraine - an updated systematic review and meta-analysis. BMC Neurol. 2020;20(1):57.

26. Charlson ME, Pompei P, Ales KL, MacKenzie CR. A new method of classifying prognostic comorbidity in longitudinal studies: development and validation. J Chronic Dis. 1987;40(5):373-83.

27. Reilly MC, Zbrozek AS, Dukes EM. The validity and reproducibility of a work productivity and activity impairment instrument. Pharmacoeconomics.1993:4(5):353-65.

28. Linde M, Gustavsson A, Stovner LJ, et al. The cost of headache disorders in Europe: the Eurolight project. Eur J Neurol. 2012;19(5):703-11.

29. Bloudek LM, Stokes M, Buse DC, et al. Cost of healthcare for patients with migraine in five European countries: results from the International Burden of Migraine Study (IBMS). J Headache Pain. 2012;13(5):361-78.

30. Lofland JH, Pizzi L, Frick KD. A review of health-related workplace productivity loss instruments. Pharmacoeconomics. 2004;22(3):165-84.

31. Xie F, Thumboo J, Fong KY, et al. A study on indirect and intangible costs for patients with knee osteoarthritis in Singapore. Value Health. 2008;11(Suppl 1):S84-S90

32. Parsons LS. Performing a 1: N case-control match on propensity score Paper presented at: Proceedings of the 29th Annual SAS Users Group International Conference; May 9-12, 2004; Montreal, Canada.

33. Dodick DW, Loder EW, Manack Adams A, et al. Assessing barriers to chronic migraine consultation, diagnosis, and treatment: results from the Chronic Migraine Epidemiology and Outcomes (CaMEO) study. Headache. 2016;56(5):821-34
34. Zwart JA, Dyb G, Hagen K, et al. Depression and anxiety disorders associated with headache frequency. The Nord-Trondelag Health Study. Eur J Neurol. 2003;10(2):147-52.

35. Buse DC, Silberstein SD, Manack AN, Papapetropoulos S, Lipton RB. Psychiatric comorbidities of episodic and chronic migraine. J Neurol. 2013;260(8):1960-69

36. Buse DC, Reed ML, Fanning KM, et al. Comorbid and co-occurring conditions in migraine and associated risk of increasing headache pain intensity and headache frequency: results of the Migraine in America Symptoms and Treatment (MAST) study. J Headache Pain. 2020;21(1):23.

37. Buse DC, Greisman JD, Baigi K, Lipton RB. Migraine progression: a systematic review. Headache. 2019;59(3):306-38.

38. Ashina S, Serrano D, Lipton RB, et al. Depression and risk of transformation of episodic to chronic migraine. J Headache Pain. 2012;13(8):615-24.

39. Pesa J, Lage MJ. The medical costs of migraine and comorbid anxiety and depression. Headache. 2004;44(6):562-70

40. Blumenfeld AM, Varon SF, Wilcox TK, et al. Disability, HRQoL and resource use among chronic and episodic migraineurs: results from the International Burden of Migraine Study (IBMS). Cephalalgia. 2011;31(3):301-15

41. Gibbs SN, Shah S, Deshpande CG, et al. United States patients' perspective of living with migraine: country-specific results from the global "My Migraine Voice" survey. Headache. May 5, 2020. Online ahead of print. Available at: https://headachejournal.onlinelibrary.wiley.com/doi/ full/10.1111/head.13829. Accessed June 16, 2020.

42. Takaki H, Onozuka D, Hagihara A. Migraine-preventive prescription patterns by physician specialty in ambulatory care settings in the United States. Prev Med Rep. 2017;9:62-67.

43. Hawkins K, Wang S, Rupnow M. Direct cost burden among insured U.S. employees with migraine. Headache. 2008;48(4):553-63.

44. Hawkins K, Wang S, Rupnow MF. Indirect cost burden of migraine in the United States. J Occup Environ Med. 2007;49(4):368-74.

45. Dodick DW. CGRP ligand and receptor monoclonal antibodies for migraine prevention: evidence review and clinical implications. Cephalalgia. 2019;39(3):445-58

46. Edvinsson L. The CGRP pathway in migraine as a viable target for therapies. Headache. 2018;58(Suppl 1):33-47.

47. Ernst MM, O'Brien HL, Powers SW. Cognitive-behavioral therapy: how medical providers can increase patient and family openness and access to evidence-based multimodal therapy for pediatric migraine. Headache. 2015;55(10):1382-96

48. Minen MT, Azarchi S, Sobolev R, et al. Factors related to migraine patients' decisions to initiate behavioral migraine treatment following a headache specialist's recommendation: a prospective observational study. Pain Med. 2018;19(11):2274-82.

49. Pérez-Muñoz A, Buse DC, Andrasik F. Behavioral interventions for migraine. Neurol Clin. 2019;37(4):789-813. 